ISSN 0258-7122 (Print), 2408-8293 (Online)

Bangladesh J. Agril. Res. 42(1): 159-170, March 2017

\title{
EFFECT OF SUBSTRATES TO FORMULATE Trichoderma harzianum BASED BIO-FUNGICIDE IN CONTROLLING SEEDLING DISEASE (Rhizoctonia solani) of BRINJAL
}

\author{
M. I. FARUK ${ }^{1}$ AND M. L. RAHMAN ${ }^{2}$
}

\begin{abstract}
Efficacy of three different substrates viz., rice bran, wheat bran, grass pea bran and their combinations with mustard oilcake (MOC) were tested to formulate a suitable Trichoderma harzianum based bio-fungicide for controlling seedling disease of brinjal caused by Rhizoctonia solani in tray soil as well as in seedbed soil under net house condition of Bangladesh Agricultural Research Institute (BARI), Gazipur during 2010 to 2014. The results of three years experiments revealed that $T$. harzianum bio-fungicides formulated in five different combinations of substrates viz., (1) rice bran + wheat bran, (2) rice bran + mustard oilcake (MOC) (3) rice bran + grasspea bran, (4) rice bran + wheat bran $+\mathrm{MOC}$ and (5) rice bran + grasspea bran $+\mathrm{MOC}$ were equally effective to control the soil borne seedling disease of brinjal caused by Rhizoctonia solani in tray soil and seedbed condition. In addition, vegetative growth of brinjal seedlings viz., shoot length, shoot weight, root length and root weight were enhanced significantly by the T. harzianum bio-fungicides in $R$. solani inoculated seedbed condition.
\end{abstract}

Keywords: Trichoderma harzianum, Rhizoctonia solani, brinjal seedling.

\section{Introduction}

Quality food and nutrition are the serious challenges worldwide where vegetable can play a vital role in everyday diet in general. Among the vegetables, brinjal (Solanum melongena L.) is the major crop that achieves tremendous popularity over the last century in Bangladesh. It grows round the year in any space available for crop cultivation in the country and uses as multifarious item of every day dish (Rashid, 1976; Bose and Som, 1986). The productivity of brinjal in Bangladesh is $17.5 \mathrm{t} / \mathrm{ha}$ while in Japan $32 \mathrm{t} / \mathrm{ha}$, Italy $28.2 \mathrm{t} / \mathrm{ha}$ and Turkey 30.2 $\mathrm{t} /$ /ha (FAOSTAT, 2012). It is estimated that $10 \%$ of crops are lost due to plant diseases worldwide annually which incurs considerable financial losses to the farmers (Strange and Scott, 2005). The pathogen Rhizoctonia solani causing germination failure, damping off and seedling rot is the major constraint to brinjal cultivation (Najar et al., 2011; Seema and Devaki, 2010). The management of this soil borne disease is difficult owing to long saprophytic survival ability of pathogen in soil (Dey, 2005). Suppression or elimination of soil borne inoculums is the only effective solution which may be achieved

${ }^{1}$ Senior Scientific Officer, Plant Pathology Division, Bangladesh Agricultural Research Institute (BARI), Gazipur, Bangladesh, ${ }^{2}$ Director, Research Wing, BARI, Gazipur, Bangladesh. 
through effective fungal antagonists. The beneficial microbe such as Trichoderma harzianum has been reported as a potential bio-control agent that effectively controlled the soil borne pathogens including $R$. solani (Elad et al., 1980; Roy et al., 1989; Anand and Reddy, 2009). The Trichoderma spp. are considered as potential biocontrol and plant growth promoting agents for many crop plants (Verma et al., 2007; Bai et al., 2008; Savazzini et al., 2009). The native bio-control agents usually remain in the agricultural soil in low population density. Augmenting their density to higher stability level in soil through artificial inoculation may successfully control soil borne pathogens in brinjal seed bed. Many bio-product/farm household bio-products which are locally available could easily be used as substrates to promote Trichoderma population in the soil. Therefore, the present study was undertaken to find out the effective local substrates to formulate the best medium for mass culturing of $T$. harzianum to be used as effective bio-fungicides against seedling disease $(R$. solani) of brinjal under seed bed condition.

\section{Materials and Method}

An experiment was conducted in the plastic tray and three other experiments in seed bed conditions of the net house of Plant Pathology Division, Bangladesh Agricultural Research Institute (BARI), Gazipur during the period from 2010 to 2014 to find out the suitable carrier material for mass culturing of Trichoderma harzianum and thereby formulation of effective bio-fungicides against Rhizoctonia solani causing seedling disease of brinjal. A pure culture of biocontrol agent $T$. harzianum (TM14) was grown on Potato Dextrose Agar (PDA) medium which was subsequently used as an inoculum of bio-fungicide that multiplied on rice bran, wheat bran, grasspea bran and their combination with mustard oilcake (MOC). The treatment combinations of the study were $\mathrm{T}_{1}=$ Rice bran, $\mathrm{T}_{2}=$ Wheat bran, $\mathrm{T}_{3}=$ Grasspea bran, $\mathrm{T}_{4}=$ Rice bran + Wheat bran $(1: 1)$, $\mathrm{T}_{5}=$ Rice bran + Grasspea bran (1:1), $\mathrm{T}_{6}=$ Rice bran + MOC (1:1), $\mathrm{T}_{7}=$ Rice bran + Wheat bran + MOC (1:1:1), $\mathrm{T}_{8}=$ Rice bran + Grasspea bran + MOC (1:1:1), $\mathrm{T}_{9}=$ Wheat bran + Grasspea bran + MOC $(1: 1: 1), \mathrm{T}_{10}=$ Rice bran + Wheat bran + Grass pea bran+ MOC (1:1:1:1), $\mathrm{T}_{11}=$ Seed treatment with Provax and $\mathrm{T}_{12}=$ Control. According to the treatment combinations $600 \mathrm{~g}$ of individual or combination of substrate materials were taken separately in $1000 \mathrm{ml}$ Erlenmeyer flasks and sterilized in an autoclave at $121^{\circ} \mathrm{C}$ for 15 minutes. After cooled down the substrate was inoculated individually with $5 \mathrm{~mm}$ diameter mycelia disc of five-day old culture of $T$. harzianum grown on PDA and finally incubated at $25 \pm 2{ }^{\circ} \mathrm{C}$ for 15 days. After incubation the colonized substrates were removed from the flasks and air dried and preserved in refrigerator at $10{ }^{\circ} \mathrm{C}$. Besides, the pathogenic fungus $R$. solani was multiplied on sterilized barley grains in $1000 \mathrm{ml}$ Erlenmeyer flask at temperature of $25 \pm 2{ }^{\circ} \mathrm{C}$ for 15 days. 
In plastic tray experiment both pathogenic fungus $R$. solani and $T$. harzianum bio-fungicide were used at the rate of @ ) $20 \mathrm{~g} / \mathrm{kg}$ soil where the control treatment received $R$. solani only and the seeds of BARI Bagun-7 were sown in the tray soil @100 seeds per tray. The experiment was laid out in completely randomized design (CRD) with six replications. Intercultural operations were done to grow the crop (Anon., 2007).

In seed bed trials the colonized barley grains were incorporated in the seed bed soils@100 g/ $\mathrm{m}^{2}$ soil. Inoculated seed bed soil was allowed to multiply the pathogen $R$. solani for 10 days with proper soil moisture. The T. harzianum biofungicides were incorporated to the seed bed and kept for 7 days with proper soil moisture for establishment of $T$. harzianum in the soils. The control bed received only $R$. solani. The seeds of BARI Bagun-7 were sown in the seed bed @ 200 seeds per treatment. The experiment was laid out in completely randomized design (CRD) with four replications. Necessary intercultural operations were done as per recommendation of the crop (Anon., 2007).

Data were collected on percent seed germination, seedling mortality, shoot height, shoot weight, root length and root weight of brinjal seedlings. The percent data were converted into arcsine transformation values before statistical analysis. The data were analyzed statistically and means were separated by Duncun's New Multiple Range Test (DMRT).

\section{Results and Discussion}

\section{Screening of substrates based T. harzianum bio-fungicides in plastic tray}

The seedling emergence of brinjal was significantly higher and ranged from $76 \%$ (grasspea bran) to $95 \%$ (rice bran + mustard oilcake) due to the carrier based on T. harzianum bio-fungicide treatments whereas untreated control tray gave lower seedling emergence (71\%) (Table 1). Pre-emergence and post-emergence seedling mortality was also varied among the treatments and the highest seedling mortality of $47 \%$ was recorded in control trays. Soil treatment with T. harzianum bio-fungicides reduced seedling mortality of $25.53 \%-76.60 \%$ as compared to untreated control (Table 1). The result showed that $T$. harzianum treated tray soil gave higher amount of healthy seedlings $(65 \%-89 \%)$ while untreated control tray soil produced only $53 \%$ healthy seedling in $R$. solani inoculated soil. The overall performance of the bio-fungicide $T$. harzianum with respect to seedling emergence, seedling mortality and healthy seedlings of brinjal under $R$. solani inoculated condition was better. 
Table 1. Effect of different carrier based $T$. harzianum bio-fungicides on the emergence and mortality of brinjal seedling in $R$. solani inoculated soils in the plastic tray

\begin{tabular}{|c|c|c|c|c|c|c|}
\hline $\begin{array}{c}\text { Name of } \\
\text { substrates for } T \text {. } \\
\text { harzianum }\end{array}$ & $\begin{array}{l}\text { Seedling } \\
\text { emergence } \\
(\%)\end{array}$ & $\begin{array}{l}\text { Pre- } \\
\text { emergence } \\
\text { mortality } \\
(\%)\end{array}$ & $\begin{array}{c}\text { Post- } \\
\text { emergence } \\
\text { seedling } \\
\text { mortality } \\
(\%)\end{array}$ & $\begin{array}{c}\text { Total } \\
\text { seedling } \\
\text { mortality } \\
(\%)\end{array}$ & $\begin{array}{l}\text { Seedling } \\
\text { mortality } \\
\text { reduction } \\
(\%) \text { over } \\
\text { control }\end{array}$ & $\begin{array}{c}\text { Total } \\
\text { healthy } \\
\text { seedling } \\
(\%)\end{array}$ \\
\hline Rice bran & 82 & 18 & 5 & 23 & 51.06 & 77 \\
\hline Wheat bran & 81 & 19 & 13 & 32 & 31.91 & 68 \\
\hline Grasspea bran & 76 & 24 & 11 & 35 & 25.53 & 65 \\
\hline $\begin{array}{l}\text { Rice bran }+ \\
\text { Wheat bran }\end{array}$ & 88 & 12 & 12 & 24 & 48.94 & 76 \\
\hline $\begin{array}{l}\text { Rice bran }+ \\
\text { Grasspea bran }\end{array}$ & 85 & 15 & 7 & 22 & 53.19 & 78 \\
\hline Rice bran + MOC & 95 & 5 & 6 & 11 & 76.6 & 89 \\
\hline $\begin{array}{l}\text { Rice bran }+ \\
\text { Wheat bran }+ \\
\text { MOC }\end{array}$ & 86 & 14 & 13 & 27 & 42.55 & 73 \\
\hline $\begin{array}{l}\text { Rice bran }+ \\
\text { Grasspea bran } \\
+ \text { MOC }\end{array}$ & 92 & 8 & 8 & 16 & 65.96 & 84 \\
\hline $\begin{array}{l}\text { Wheat bran }+ \\
\text { Grasspea bran }+ \\
\text { MOC }\end{array}$ & 85 & 15 & 8 & 23 & 51.06 & 77 \\
\hline $\begin{array}{l}\text { Rice bran }+ \\
\text { Wheat bran }+ \\
\text { Grasspea bran }+ \\
\text { MOC }\end{array}$ & 91 & 9 & 8 & 17 & 63.83 & 83 \\
\hline $\begin{array}{l}\text { Seed treatment } \\
\text { with Provax }\end{array}$ & 86 & 14 & 11 & 35 & 25.53 & 65 \\
\hline Untreated Control & 71 & 29 & 18 & 47 & - & 53 \\
\hline
\end{tabular}

Efficacy of substrates based $T$. harzianum bio-fungicides and provax in seed bed

The emergence of brinjal seedling in $R$. solani inoculated soils was sharply enhanced by the application of different carrier material based $T$. harzianum biofungicides and Provax in seedbed (Table 2). The individual as well as combined application of $T$. harzianum bio-fungicides was found superior in seedling emergence as compared to the untreated control during three consecutive years. The seedling emergence of brinjal ranged from $55.67 \%$ to $65.67 \%, 71.67 \%$ to $80.67 \%$, and $60 \%$ to $72 \%$, respectively in consecutive three years due to the 
application of different bio-fungicides in seed bed while lower seedling emergence $(49.33 \%, 56.67 \%$, and $50 \%)$ was recorded from the control seedbed. The trend of pre-emergence mortality was almost similar among the biofungicide treatments over the years that ranged from $39.68-44.33 \%$ in $1^{\text {st }}$ year trial, $19.33-28.33 \%$ in $2^{\text {nd }}$ year and $28-40 \%$ in $3^{\text {rd }}$ year trial (Table 2 ). The preemergence seedling mortality in the control treatments was 50.67, 43.33, and $50 \%$ during $1^{\text {st }}, 2^{\text {nd }}$ and $3^{\text {rd }}$ year trials, respectively. The effect of Provax was inferior or similar to that of T. harzianum bio-fungicide in respect of emergence and mortality of brinjal seedling in seedbed. The results of three years trial indicated that the effects of single as well as mixed carrier based T. harzianum bio-fungicides were very much similar in increasing seedling emergence and reducing pre-emergence mortality of brinjal seedling caused by $R$. solani under seedbed conditions.

Table 2. Effect of different carrier material based $T$. harzianum bio-fungicides on the emergence and pre-emergence mortality of brinjal seedling in $R$. solani inoculated seed bed soil

\begin{tabular}{l|ccc|cccc}
\hline \multirow{2}{*}{ Name of substrates } & \multicolumn{3}{|c|}{$\begin{array}{c}\text { Seedling emergence of brinjal } \\
\text { in consecutive three years (\%) }\end{array}$} & \multicolumn{3}{c}{$\begin{array}{c}\text { Pre-emergence mortality in } \\
\text { consecutive three years (\%) }\end{array}$} \\
\cline { 2 - 8 } & $1^{\text {st } y e a r}$ & $2^{\text {nd }}$ year & $3^{\text {rd }}$ year & $1^{\text {st } y e a r}$ & $2^{\text {nd }}$ year & $3^{\text {rd }}$ year \\
\hline Rice bran & 59.00 & 77.67 & 60.00 & 41.00 & 22.33 & 40.00 \\
Wheat bran & 59.33 & 74.33 & 61.00 & 40.67 & 25.67 & 39.00 \\
Grasspea bran & 56.00 & 71.67 & 69.00 & 44.00 & 28.33 & 31.00 \\
$\begin{array}{l}\text { Rice bran + Wheat bran } \\
\text { Rice bran + Grass pea bran }\end{array}$ & 60.33 & 74.33 & 67.00 & 39.68 & 25.67 & 33.00 \\
$\begin{array}{l}\text { Rice bran + Mustard } \\
\text { oilcake }\end{array}$ & 55.67 & 75.00 & 67.00 & 42.33 & 25.00 & 33.00 \\
$\begin{array}{l}\text { Rice bran + Wheat bran + } \\
\text { MOC }\end{array}$ & 58.00 & 79.67 & 69.00 & 44.33 & 19.33 & 31.00 \\
$\begin{array}{l}\text { Rice bran + Grasspea bran } \\
+ \text { MOC }\end{array}$ & 57.67 & 76.00 & 70.00 & 42.00 & 20.33 & 32.00 \\
$\begin{array}{l}\text { Wheat bran + Grass pea } \\
\text { bran + MOC }\end{array}$ & 57.33 & 74.33 & 72.00 & 42.67 & 25.67 & 28.00 \\
$\begin{array}{l}\text { Wheat bran + Grass pea } \\
\text { bran+ Rice bran + MOC }\end{array}$ & 65.67 & 72.33 & 66.00 & 44.33 & 27.67 & 34.00 \\
$\begin{array}{l}\text { Seed treatment with Provax } \\
\text { Control }\end{array}$ & 63.67 & 77.67 & 59.00 & 46.33 & 22.33 & 41.00 \\
\hline
\end{tabular}

The post emergence seedling mortality of brinjal in $R$. solani inoculated soils in seed bed was significantly reduced by the application of different carrier material based T. harzianum bio-fungicides and Provax (Table 3). The individual as well as combination of mixed carrier material based $T$. harzianum bio-fungicides were found superior in reduction of post emergence seedling mortality as compared to 
the untreated control. The highest seedling mortality of $17.33 \%, 25.33 \%$ and $21 \%$, respectively was recorded in untreated control seedbed during the consecutive three years. Seedling mortality of brinjal ranged from 4.67\%-7.33\% in $1^{\text {st }}$ year, $6.67 \%-12.67 \%$ in $2^{\text {nd }}$ year and $7.67 \%-9.33 \%$ in $3^{\text {rd }}$ year due to the application of $T$. harzianum bio-fungicides were recorded (Table 3). Provax treated seedbed showed similar seedling mortality as observed in the biofungicides treated beds every year. The effect of different carrier material based T. harzianum bio-fungicides was much encouraging that reduced post-emergence seedling mortality of brinjal from $57.70 \%-73.05 \%$ in $1^{\text {st }}$ year, $49.98 \%-73.67 \%$ in $2^{\text {nd }}$ year and $55.57 \%-63.48 \%$ in $3^{\text {rd }}$ year over untreated control in $R$. solani inoculated seedbeds (Table 3 ).

Table 3. Efficacy of various carrier material based $T$. harzianum bio-fungicides on the post-emergence mortality of brinjal seedling in $R$. solani inoculated soils in seed bed

\begin{tabular}{|c|c|c|c|c|c|c|}
\hline \multirow[t]{2}{*}{ Name of substrates } & \multicolumn{3}{|c|}{$\begin{array}{l}\text { Post-emergence seedling mortality } \\
\text { in consecutive three years }(\%)\end{array}$} & \multicolumn{3}{|c|}{$\begin{array}{l}\text { Seedling mortality reduction } \\
\text { over control in consecutive } \\
\text { three years }(\%)\end{array}$} \\
\hline & $1^{\text {st }}$ year & $2^{\text {nd }}$ year & $3^{\text {rd }}$ year & $1^{\text {st }}$ year & $2^{\text {nd }}$ year & $3^{\text {rd }}$ year \\
\hline Rice bran & $\begin{array}{l}7.00 \mathrm{bc} \\
(15.31)\end{array}$ & $\begin{array}{l}12.67 \mathrm{~b} \\
(20.84)\end{array}$ & $\begin{array}{l}9.33 \mathrm{~b} \\
(17.05)\end{array}$ & 59.61 & 49.98 & 55.57 \\
\hline Wheat bran & $\begin{array}{l}6.33 \mathrm{bc} \\
(14.58)\end{array}$ & $\begin{array}{l}10.00 \mathrm{~b} \\
(17.71)\end{array}$ & $\begin{array}{c}9.00 \mathrm{~b} \\
(17.44)\end{array}$ & 63.47 & 60.52 & 57.14 \\
\hline Grasspea bran & $\begin{array}{l}7.33 \mathrm{~b} \\
(15.72)\end{array}$ & $\begin{array}{l}11.00 \mathrm{~b} \\
(19.36)\end{array}$ & $\begin{array}{l}9.33 \mathrm{~b} \\
(16.75)\end{array}$ & 57.70 & 56.57 & 55.57 \\
\hline Rice bran + Wheat bran & $\begin{array}{l}5.67 \mathrm{bc} \\
(13.77)\end{array}$ & $\begin{array}{l}10.67 \mathrm{~b} \\
(18.89)\end{array}$ & $\begin{array}{c}7.67 \mathrm{~b} \\
(16.05)\end{array}$ & 67.28 & 57.88 & 63.48 \\
\hline $\begin{array}{l}\text { Rice bran + Grasspea } \\
\text { bran }\end{array}$ & $\begin{array}{l}5.33 \mathrm{bc} \\
(13.36)\end{array}$ & $\begin{array}{l}8.00 \mathrm{~b} \\
(16.41)\end{array}$ & $\begin{array}{l}8.33 \mathrm{~b} \\
(16.75)\end{array}$ & 69.24 & 68.42 & 60.33 \\
\hline $\begin{array}{l}\text { Rice bran + Mustard } \\
\text { oilcake }\end{array}$ & $\begin{array}{l}6.00 \mathrm{bc} \\
(14.19)\end{array}$ & $\begin{array}{l}8.67 \mathrm{~b} \\
(17.05)\end{array}$ & $\begin{array}{l}9.00 \mathrm{~b} \\
(16.75)\end{array}$ & 65.38 & 65.77 & 57.14 \\
\hline $\begin{array}{l}\text { Rice bran + Wheat bran } \\
+ \text { MOC }\end{array}$ & $\begin{array}{l}5.33 \mathrm{bc} \\
(13.38)\end{array}$ & $\begin{array}{l}6.67 \mathrm{~b} \\
(14.95)\end{array}$ & $\begin{array}{l}8.67 \mathrm{~b} \\
(16.02)\end{array}$ & 69.24 & 73.67 & 58.71 \\
\hline $\begin{array}{l}\text { Rice bran +Grasspea } \\
\text { bran +MOC }\end{array}$ & $\begin{array}{l}5.33 \mathrm{bc} \\
(13.41)\end{array}$ & $\begin{array}{l}9.00 \mathrm{~b} \\
(17.21)\end{array}$ & $\begin{array}{l}8.33 \mathrm{~b} \\
(17.08)\end{array}$ & 69.24 & 64.47 & 60.33 \\
\hline $\begin{array}{l}\text { Wheat bran + Grasspea } \\
\text { bran + MOC }\end{array}$ & $\begin{array}{l}7.00 \mathrm{bc} \\
(15.33)\end{array}$ & $\begin{array}{l}9.00 \mathrm{~b} \\
(17.39)\end{array}$ & $\begin{array}{l}9.33 \mathrm{~b} \\
(17.78)\end{array}$ & 59.61 & 64.47 & 55.57 \\
\hline $\begin{array}{l}\text { Wheat bran + Grasspea } \\
\text { bran+ Rice bran }+ \\
\text { MOC }\end{array}$ & $\begin{array}{c}4.67 \mathrm{c} \\
(12.48)\end{array}$ & $\begin{array}{c}8.67 \mathrm{~b} \\
(16.96)\end{array}$ & $\begin{array}{c}9.00 \mathrm{~b} \\
(16.77)\end{array}$ & 73.05 & 65.77 & 57.14 \\
\hline $\begin{array}{l}\text { Seed treatment with } \\
\text { Provax }\end{array}$ & $\begin{array}{l}6.00 \mathrm{bc} \\
(14.21)\end{array}$ & $\begin{array}{l}9.33 \mathrm{~b} \\
(17.49)\end{array}$ & $\begin{array}{l}8.33 \mathrm{~b} \\
(17.75)\end{array}$ & 65.38 & 63.17 & 60.33 \\
\hline Control & $\begin{array}{l}17.33 \mathrm{a} \\
(24.59)\end{array}$ & $\begin{array}{l}25.33 \mathrm{a} \\
(30.17)\end{array}$ & $\begin{array}{l}21.00 \mathrm{a} \\
(27.48)\end{array}$ & - & - & - \\
\hline
\end{tabular}

Values in a column having same letter did not differ significantly ( $\mathrm{p}=0.05)$ by LSD.

Arcsine transformed values were within the parentheses. 
Shoot length and shoot weight of seedlings were increased significantly $(\mathrm{p}=0.05)$ by the $T$. harzianum bio-fungicides in the $R$. solani inoculated seed bed soil (Table 4). In $1^{\text {st }}$ year trial, the shoot length of brinjal seedlings ranged from 15.87 $\mathrm{cm}$ to $18.80 \mathrm{~cm}$ due to $T$. harzianum bio-fungicides and minimum shoot length $(13.40 \mathrm{~cm})$ was obtained from the control bed. Similarly, application of individual and mixed carrier material based T. harzianum bio-fungicides gave higher shoot length ranged from $7.67 \mathrm{~cm}-10.60 \mathrm{~cm}$ in $2^{\text {nd }}$ year and $6.77 \mathrm{~cm}$ $9.07 \mathrm{~cm}$ in $3^{\text {rd }}$ year, while shorter shoots were observed in Provax and untreated control seedbed (Table 4). The shoot weight of individual brinjal seedling was enhanced up to $8.72 \mathrm{~g}$ by the T. harzianum bio-fungicide where minimum shoot weight of $5.87 \mathrm{~g}$ was recorded from the control bed during the $1^{\text {st }}$ year trial. Similarly, maximum shoot weights of $6.80 \mathrm{~g}$, and $7.80 \mathrm{~g}$ were recorded from the bio-fungicide treated seedbeds of $2^{\text {nd }}$ and $3^{\text {rd }}$ year trials where control beds gave shoots of $3.28 \mathrm{~g}$ and $4.37 \mathrm{~g}$, respectively. Seed treatment with chemical fungicide Provax gave comparatively lower shoot weight of brinjal seedling in all the years.

Table4. Effect of different carrier material based T. harzianum bio-fungicides on the shoot growth of brinjal seedling in $R$. solani inoculated seed bed soil

\begin{tabular}{|c|c|c|c|c|c|c|}
\hline \multirow[t]{2}{*}{ Name of substrates } & \multicolumn{3}{|c|}{$\begin{array}{c}\text { Shoot length in consecutive three } \\
\text { years }(\mathrm{cm})\end{array}$} & \multicolumn{3}{|c|}{$\begin{array}{l}\text { Shoot weight in consecutive } \\
\left.\text { three years } \text { (gplant }^{-1}\right)\end{array}$} \\
\hline & $1^{\text {st }}$ year & $2^{\text {nd }}$ year & $3^{\text {rd }}$ year & $1^{\text {st }}$ year & $2^{\text {nd }}$ year & $3^{\text {rd }}$ year \\
\hline Rice bran & $16.43 \mathrm{~cd}$ & $8.07 \mathrm{bcd}$ & $9.07 \mathrm{a}$ & $8.34 \mathrm{ab}$ & $5.05 \mathrm{c}$ & $6.17 \mathrm{~b}$ \\
\hline Wheat bran & $16.80 \mathrm{bcd}$ & $8.13 \mathrm{bcd}$ & $6.77 \mathrm{bc}$ & $8.69 \mathrm{ab}$ & $4.87 \mathrm{~cd}$ & $6.60 \mathrm{~b}$ \\
\hline Grasspea bran & $17.27 \mathrm{bc}$ & $7.67 \mathrm{~cd}$ & $7.73 \mathrm{ab}$ & $8.57 \mathrm{ab}$ & $4.87 \mathrm{~cd}$ & $6.70 \mathrm{~b}$ \\
\hline Rice bran + Wheat bran & $15.87 \mathrm{~cd}$ & $8.40 \mathrm{bc}$ & $8.90 \mathrm{a}$ & $8.36 \mathrm{ab}$ & $6.76 \mathrm{a}$ & $7.80 \mathrm{a}$ \\
\hline $\begin{array}{l}\text { Rice bran }+ \text { Grasspea } \\
\text { bran }\end{array}$ & $18.80 \mathrm{a}$ & $8.22 \mathrm{bcd}$ & $8.60 \mathrm{a}$ & $8.72 \mathrm{a}$ & $6.80 \mathrm{a}$ & $7.80 \mathrm{a}$ \\
\hline $\begin{array}{l}\text { Rice bran }+ \text { Mustard } \\
\text { oilcake }\end{array}$ & $16.67 \mathrm{bcd}$ & $8.97 \mathrm{bc}$ & $8.57 \mathrm{a}$ & $8.53 \mathrm{ab}$ & $6.10 \mathrm{ab}$ & $7.73 \mathrm{a}$ \\
\hline $\begin{array}{l}\text { Rice bran + Wheat bran } \\
+ \text { MOC }\end{array}$ & $16.20 \mathrm{~cd}$ & $9.27 \mathrm{ab}$ & $8.97 \mathrm{a}$ & $8.31 \mathrm{ab}$ & $6.63 \mathrm{a}$ & $7.50 \mathrm{a}$ \\
\hline $\begin{array}{l}\text { Rice bran+ Grasspea } \\
\text { bran }+ \text { MOC }\end{array}$ & $17.03 \mathrm{bc}$ & $10.47 \mathrm{a}$ & $8.73 \mathrm{a}$ & $8.35 \mathrm{ab}$ & $6.52 \mathrm{a}$ & $7.70 \mathrm{a}$ \\
\hline $\begin{array}{l}\text { Wheat bran + Grasspea } \\
\text { bran + MOC }\end{array}$ & $16.67 \mathrm{bcd}$ & $9.43 \mathrm{ab}$ & $7.83 \mathrm{ab}$ & $8.45 \mathrm{ab}$ & $5.35 \mathrm{bc}$ & $7.43 \mathrm{a}$ \\
\hline $\begin{array}{l}\text { Wheat bran + Grasspea } \\
\text { bran+ Rice bran + } \\
\text { MOC }\end{array}$ & $18.00 \mathrm{ab}$ & $10.60 \mathrm{a}$ & $8.63 \mathrm{a}$ & $8.16 \mathrm{ab}$ & $5.10 \mathrm{c}$ & $7.77 \mathrm{a}$ \\
\hline $\begin{array}{l}\text { Seed treatment with } \\
\text { Provax }\end{array}$ & $15.33 \mathrm{~d}$ & $6.86 \mathrm{de}$ & $5.97 \mathrm{~cd}$ & $7.51 \mathrm{~b}$ & $4.19 \mathrm{~d}$ & $5.43 \mathrm{c}$ \\
\hline Control & $13.40 \mathrm{e}$ & $5.80 \mathrm{e}$ & $5.00 \mathrm{~d}$ & $5.87 \mathrm{c}$ & $3.28 \mathrm{e}$ & $4.37 \mathrm{~d}$ \\
\hline
\end{tabular}

Values in a column having same letter did not differ significantly ( $\mathrm{p}=0.05)$ by LSD. 
The root length was enhanced up to $6.80 \mathrm{~cm}$ by wheat bran based bio-fungicide followed by rice bran + grass pea bran $(6.73 \mathrm{~cm})$ and the shortest root was found in untreated control in $1^{\text {st }}$ year trials (Table 5). The root length ranged from 6.80 $\mathrm{cm}-8.60 \mathrm{~cm}$ in $2^{\text {nd }}$ year and $6.60 \mathrm{~cm}-7.93 \mathrm{~cm}$ in $3^{\text {rd }}$ year trials while the shorter roots were recorded from Provax and untreated control treatments. The $T$. harzianum based bio-fungicides also augmented the root weights of brinjal seedlings as compared to untreated control.

Table 5. Role of various carrier material based $T$. harzianum bio-fungicides on the root growth of brinjal seedling in $\boldsymbol{R}$. solani inoculated seed bed soil

\begin{tabular}{|c|c|c|c|c|c|c|}
\hline \multirow{2}{*}{ Name of substrates } & \multicolumn{3}{|c|}{$\begin{array}{l}\text { Root length in consecutive } \\
\text { three years }(\mathrm{cm})\end{array}$} & \multicolumn{3}{|c|}{$\begin{array}{l}\text { Root weight in consecutive } \\
\text { three years }\left(\text { mgplant }^{-1}\right)\end{array}$} \\
\hline & $1^{\text {st }}$ year & $2^{\text {nd }}$ year & $3^{\text {rd }}$ year & $1^{\text {st }}$ year & $2^{\text {nd }}$ year & $3^{\text {rd }}$ year \\
\hline Rice bran & $6.57 \mathrm{ab}$ & $7.00 \mathrm{abc}$ & $6.73 c$ & 400 & $440 \mathrm{a}$ & $430 \mathrm{bc}$ \\
\hline Wheat bran & $6.80 \mathrm{a}$ & $7.13 \mathrm{abc}$ & $6.87 \mathrm{bc}$ & 350 & $410 \mathrm{ab}$ & $450 \mathrm{abc}$ \\
\hline Grasspea bran & $6.57 \mathrm{ab}$ & $6.80 \mathrm{abc}$ & $6.60 \mathrm{c}$ & 390 & $410 \mathrm{ab}$ & $460 \mathrm{abc}$ \\
\hline Rice bran + Wheat bran & $6.40 \mathrm{ab}$ & $7.13 \mathrm{abc}$ & $7.93 \mathrm{a}$ & 380 & $420 \mathrm{ab}$ & $520 \mathrm{ab}$ \\
\hline $\begin{array}{l}\text { Rice bran + Grasspea } \\
\text { bran }\end{array}$ & $6.73 \mathrm{a}$ & $7.80 \mathrm{ab}$ & $7.37 \mathrm{abc}$ & 400 & $430 \mathrm{ab}$ & $500 \mathrm{abc}$ \\
\hline $\begin{array}{l}\text { Rice bran }+ \text { Mustard } \\
\text { oilcake }\end{array}$ & $6.30 \mathrm{ab}$ & $8.47 \mathrm{a}$ & $7.60 \mathrm{ab}$ & 390 & $450 \mathrm{a}$ & $520 \mathrm{ab}$ \\
\hline $\begin{array}{l}\text { Rice bran+ Wheat bran }+ \\
\text { MOC }\end{array}$ & $6.43 \mathrm{ab}$ & $8.00 \mathrm{ab}$ & $7.70 \mathrm{a}$ & 400 & $440 \mathrm{a}$ & $550 \mathrm{a}$ \\
\hline $\begin{array}{l}\text { Rice bran+ Grasspea bran } \\
+ \text { MOC }\end{array}$ & $6.50 \mathrm{ab}$ & $8.60 \mathrm{a}$ & $7.73 \mathrm{a}$ & 380 & $440 \mathrm{a}$ & $480 \mathrm{abc}$ \\
\hline $\begin{array}{l}\text { Wheat bran + Grasspea } \\
\text { bran + MOC }\end{array}$ & $6.30 \mathrm{ab}$ & $8.40 \mathrm{a}$ & $7.67 \mathrm{a}$ & 390 & $450 \mathrm{a}$ & $490 \mathrm{abc}$ \\
\hline $\begin{array}{l}\text { Wheat bran + Grasspea } \\
\text { bran+ Rice bran + MOC }\end{array}$ & $6.70 \mathrm{a}$ & $8.57 \mathrm{a}$ & $7.63 \mathrm{ab}$ & 400 & $430 \mathrm{ab}$ & $500 \mathrm{abc}$ \\
\hline $\begin{array}{l}\text { Seed treatment with } \\
\text { Provax }\end{array}$ & $5.80 \mathrm{bc}$ & $5.73 \mathrm{c}$ & $5.53 \mathrm{~d}$ & 370 & $370 \mathrm{bc}$ & $420 \mathrm{bc}$ \\
\hline Control & $5.13 \mathrm{c}$ & $5.53 \mathrm{c}$ & $4.87 \mathrm{~d}$ & 330 & $320 \mathrm{c}$ & $380 \mathrm{c}$ \\
\hline
\end{tabular}

Values in a column having same letter did not differ significantly ( $\mathrm{p}=0.05$ ) by LSD.

The overall effects of the carrier based $T$. harzianum bio-fungicides on the emergence, mortality, growth and development of brinjal seedlings under $R$. solani inoculated soils as revealed from the results of three years trials indicated that five different combination of carriers materials viz. (1) rice bran + wheat bran, (2) rice bran + grass pea bran (3) rice bran + MOC, (4) rice bran + wheat bran + MOC, and (5) rice bran + grass pea bran +MOC were superior for brinjal seedling disease ( $R$. solani) management in seed bed condition. 
Results of the present study revealed that $T$ harzianum bio-fungicides were effective to control seedling disease of brinjal caused by $R$. solani in the tray and in seedbed condition. Many researchers reported that soil amendment with formulated Trichoderma was effective in controlling seedling disease of many crops especially the fungal pathogens such as $R$. solani, Fusarium oxysporum and S. rolfsii (Lo et al., 1996; Tran, 1998; Bari et al., 2000; Shamsuzzaman et al., 2003; Benítez et al., 2004; Clear and Valic, 2005; Ngo et al., 2006; Shalini et al., 2006; Dubey et al., 2007; Rojo et al., 2007). The effect of Trichoderma spp. as bio-control agents against phytopathogenic fungi Rhizoctonia spp. and Fusarium spp. was also reported by other investigators (Poddar et al., 2004; Rojo et al., 2007). Harman et al. (2004) reported that the biocontrol agents Trichoderma spp. influenced seed germination, seedling vigor and increased shoot and root growth as well as productivity of brinjal. The growth promotion in plant with special reference to the length and weight of their shoots and roots was enhanced due to Trichoderma spp. soil amendment (Samolski et al., 2012; Harman et al., 2012; Hermosa et al., 2012). Similarly, enhanced root length and root weight of many plants were also reported by the application of Trichoderma spp. (Chang et al., 1986; Kleifeld and Chet, 1992; Azarmi et al., 2011). Elad et al. (1980) reported that incorporation of $T$. harzianum bio-fungicide in the pathogen-infested soils significantly reduced bean seedling diseases caused by $R$. solani and Sclerotium rolfsii. Meah et al. (2004) reported the formulated T. harzianum grown on peat soil based black bran was found to be effective in controlling some of the nursery diseases like damping off, tip over and seedling blight of eggplant and also promoted seed germination. The present study revealed that rice bran based Trichoderma bio-fungicide gave better seed germination, reduced seedling mortality and increased growth of brinjal seedling. Sangeetha et al. (1993) found rice bran as the best substrate for the formulation of Trichoderma which gave $35 \%$ higher seed germination in brinjal and wheat. The disease incidence of brinjal, water melon and cotton was reported to be reduced considerably by the application of T. harzianum (Sivan and Chet, 1986). Tehroni and Nazari (2004) observed T. harzianum as an effective remedy against damping-off of cucumber. Shoresh et al. (2005) stated Trichoderma spp. as effective bio-control agents for a number of soil borne plant pathogens and induced a potentate state in the plant enabling it to be more resistant to subsequent pathogen infection.

\section{Acknowledgement}

The authors gratefully acknowledge BAS-USDA for providing financial support and Bangladesh Agricultural Research Institute, Gazipur for extending necessary logistic support for smooth running of this research. Thanks are extended to the Scientific Assistant Mr. Md. Abdur Razzak and Mr. Zamil Akter for their sincere assistance in this research work. 


\section{References}

Anand, S. and J. Reddy. 2009. Biocontrol potential of Trichoderma sp. against plant pathogens, International J. of Agriculture Sci. 1: 2 Pp. 30-39.

Anonymous .2007. Research Report on Horticultural Crops (2006-07) (Pp. 93). Horticulture Research Centre, BARI, Joydebpur, Gazipu.

Azarmi, R., B. Hajieghrari and A. Giglou. 2011. Effect of Trichoderma isolates on tomato seedling growth response and nutrient uptake. African J. Biotechnology. 10(31): 5850-5855.

Bai, Z., B. Jin, Y. Li, J. Chen and Z. Li. 2008. Utilization of winery wastes for Trichoderma viride biocontrol agent production by solid state fermentation. $J$. Environ. Sci. 20: 353-358.

Bari, M.A., M. L. Rahman and I. H. Mian. 2000. Biological control of potato black scurf disease through fungal antagonist. Bangladesh J. Plant Pathol. 16(1/2): 5-7.

Benítez, T., A. M. Rincón, M. C. Limón and A. C. Codón. 2004. Biocontrol mechanisms of Trichoderma strains. International Microbiology. 7: 249-260.

Bose, T. K. and M. G. Som. 1986. Vegetables of India, Nayaprakash Calcutta, India, 63 p.

Celar, F. and N. Valic. 2005. Effects of Trichoderma spp and Glicladium roseum culture filtrates on seed germination of vegetables and maize. J. Plant Diseases \& Protect. 112(4): 343-350.

Chang, Y. C., Y. C. Chang, R. Baker, O. Kleifeld and I. Chet. 1986. Increased growth of plants in the presence of the biological control agent Trichoderma harzianum. Plant Disease. 70: 145-148.

Dey T. K. 2005. Effect of soil solarization in controlling damping-off disease of true potato seedlings. Bangladesh J. Plant pathol. 21(1\&2): 93.

Dubey, S. C., M. Suresha and B. Singha. 2007. Evaluation of Trichoderma species against Fusarium oxysporum f. sp. ciceris for integrated management of chickpea wilt. Biological Control. 40: 118-127.

Elad, Y., I. Chet and J. Katan. 1980. Trichoderma harzianum: A biocontrol agent effective against Sclerotium rolfsii and Rhizoctonia solani. Phytopathology. 70(2): 119-121.

FAOSTAT. 2012. Food and Agriculture Organization of United Nations statistical database. Retrieved from http://faostat.fao.org/.

Harman, G. E., A. H. Herrera-Estrella, B. A. Horwitz and M. Lorito. 2012. Special issue: Trichoderma - from basic biology to biotechnology. Microbiology. 158: 1-2

Harman, G. E., C. R. Howell, A. Viterbo, I. Chet and M. Lorito. 2004. Trichoderma species opportunistic, avirulent plant symbionts. Nature Reviews Microbiology. 2(1): 43-56.

Hermosa, R., A. Viterbo, I. Chet and E. Monte. 2012. Plant-beneficial effects of Trichoderma and of its genes. Microbiology. 158: 17-25 
Kleifeld, O. and I. Chet. 1992. Trichoderma-plant interaction and its effect on increased growth response. Plant \& Soil. 144: 267-272.

Lo, C. T., E. B. Nelson and G. E. Harman. 1996. Biological control of turfgrass diseases with a rhizosphere competent strains Trichoderma harzianum. Plant Disease. 80(7): 736-741.

Meah, M. B., M. R. Islam and M. M. Islam. 2004. Development of an integrated approach for management of Phomopsis Blight and Fruit rot of eggplant in Bangladesh (pp.62). Annual Research Report. Department of Plant Pathology, BAU, Mymensingh, Bangladesh.

Najar, A.G., A. Anwar, L. Masoodi and M. S. Khar. 2011. Evaluation of native biocontrol agents against Fusarium solani f. sp. melongenae causing wilt disease of brinjal in Kashmir. J. Phytopathology. 3: 31-34.

Ngo, B. H., D. N. Vu and D. Q. Tran. 2006. Analyze antagonist effects of Trichoderma spp. for controlling southern stem rot caused by Sclerotium rolfsii on peanut. Plant Protection. 1: 12-14.

Poddar, R. K., D.V. Singh and S.C. Dubey. 2004. Integrated application of Trichoderma harzianum mutants and carbendazim to manage chickpea wilt (Fusarium oxysporum f.sp. ciceri). Indian J. Agric. Sci. 74: 346-348.

Rashid, M. M. 1976. Bangladesher Sabji. Ist Edn., Bangla Academy, Dacca. 72-73 pp.

Rojo, F. G., M. M. Reynoso, M. F. Sofia, N. Chulze1 and A.M. Torres. 2007. Biological control by Trichoderma species of Fusarium solani causing peanut brown root rot under field conditions. Crop Protection. 26: 549-555.

Roy, S. J., B. S. Das and L. C. Bora. 1989. Non pestcidal management of damping-off of cabbage caused by Rhizoctonia solani kuehn. J. Agril. Sci. Society of North-East India. 11(2): 127-130.

Samolski, I., A. M. Rincon , L. M. Pinzon, A.Viterbo and E. Monte. 2012. The qid74 gene from Trichoderma harzianum has a role in root architecture and plant biofertilization. Microbiology. 158: 129-138.

Sangeetha, P., R. Jeyarajan and S. Panicker. 1993. Mass multiplication of bio-control agent Trichoderma spp. Indian Journal of Mycology and Plant Pathol. 23(3): 328330 .

Savazzini, F., C. M. O. Longa and I. Pertot. 2009. Impact of the biocontrol agent Trichoderma atroviride SC1 on soil microbial communities of a vineyard in northern mycoparasite antagonism by Trichoderma harzianum. J. Bacteriology. 178: 63826385.

Seema, M. and N. S. Deva ki. 2010. Effect of some essential oils on Rhizoctonia solani Kuhn infecting flue-cured Virginia tobacco. J. of Biopesticides. 3(3): 563-566.

Shalini, K. P., L. Narayan and A. S. Kotasthane. 2006. Genetic relatedness among Trichoderma isolates inhibiting a pathogenic fungi Rhizoctonia solani, African J. Biotechnology. 5(8): 580-584. 
Shamsuzzaman, S. M. A. Islam and I. Hossain. 2003. Trichoderma culture and germination of sweet gourd seed. Bangladesh J. Seed Sci. \& Tech. 7(1/2): 91-95.

Shoresh, M., I. Yedidia and I. Chet. 2005. Involvement of Jasmonic Acid/Ethylene Signaling Pathway in the Systemic Resistance Induced in Cucumber by Trichoderma asperellum $\mathrm{T}_{203}$. Phytopathology. 95(1): 76-84.

Sivan, A., and I. Chet. 1986. Biological control of Fusarium spp. in cotton, wheat and muskmelon by Trichoderma harzianum. J. Phytopathology. 116(1): 39-47.

Strange, R. N. and P. R. Scott. 2005. Plant disease: a threat to global food security. Annual Review of Phytopathology. 43: 83-116.

Tehroni, A. S. and S. Nazari. 2004. Antagonistic effects of Trichoderma harzianum on Phytophthora derchsleri, the causal agent of cucumber damping off. Acta horticulturae. 635: 137-139.

Tran, T. T. 1998. Antagonistic effectiveness of Trichoderma against plant fungal pathogens. Plant Protection. 4: 35-38.

Verma, M., S. K. Brar, R. D. Tyagi, V. Sahai, D. Prévost, J. R. Valéro and R. Y. Surampalli 2007. Bench-scale fermentation of Trichoderma viride on wastewater sludge: rheology, lytic enzymes and biocontrol activity. Enzyme Microbiology Technology. 41: 764-771. 